HNO 2005 · 53:681

DOI 10.1007/s00106-005-1296-0

Online publiziert: 14. Juli 2005

๑) Springer Medizin Verlag 2005

H.-P. Zenner • Universitäts-HNO-Klinik Tübingen

\title{
Schwerpunkt Forschung
}

ie vorliegende Ausgabe der HNO präsentiert sich in ungewöhnlicher Form, nämlich ausschließlich mit Originalarbeiten bestückt. Üblicherweise ist es gerade die Vielfalt der Rubriken, die die HNO für eine Leserschaft mit unterschiedlichsten Interessenschwerpunkten attraktiv macht. Die Zugkraft der HNO ist jedoch mittlerweile so stark, dass laufend zahlreiche exzellente Originalien eingereicht werden, die unbedingt in einer angemessenen Zeitspanne veröffentlicht werden sollten, damit die Ergebnisse nicht bei Drucklegung bereits veraltet sind. Wir haben deshalb in dieser Ausgabe der HNO ausnahmsweise auf die anderen Sparten verzichtet, um Klinikern und Forschern aus der HNO-Heilkunde die Gelegenheit zu bieten, ihre aktuellen Forschungsergebnisse zeitnah den Lesern vorstellen zu können.

Ich hoffe, dass uns mit der Themenzusammenstellung eine Mischung gelungen ist, die den Bedürfnissen unserer Leser entspricht.

Ihr

\section{Aans teunw}

H.-P. Zenner

\section{Korrespondierender Autor}

\section{Prof. H.-P. Zenner}

Universitäts-HNO-Klinik,

Elfriede-Aulhorn-Straße 5, 72076 Tübingen

E-Mail:zenner@uni-tuebingen.de

Interessenkonflikt: Der korrespondierende Autor versichert, dass keine Verbindungen mit einer Firma, deren Produkt in dem Artikel genannt ist, oder einer Firma, die ein Konkurrenzprodukt vertreibt, bestehen. 